\title{
Development and optimisation of micromechanical testing techniques to study the properties of meniscal tissue
}

\author{
Jared Maritz $^{a}$, Francesca Murphy ${ }^{a}$, Kalin Dragnevski ${ }^{a} \&$ Olga Barrera ${ }^{b, c, d}$ \\ ${ }^{a}$ Department of Engineering Science, University of Oxford, Parks Road, Oxford, OX1 3PJ \\ ${ }^{b}$ SnT, University of Luxembourg, ${ }^{c}$ NDORMS, University of Oxford, ${ }^{d}$ School of Engineering, computing and Mathematics, Oxford Brookes \\ University
}

\begin{abstract}
In this paper we present the results from a recent micromechanical investigation aimed at developing methodologies for testing and understanding the fundamental behaviour of meniscal tissue. To achieve this, we employed two distinctly different, but equally relevant mechanical testing platforms - uniaxial tensile testing and Dynamic Mechanical Analysis. The results from the tensile tests revealed that the studied material exhibits non-linear stress-strain behaviour and that its viscoelastic properties are timedependent. Furthermore, by using DMA it was possible to perform walking and running simulations, which provided further information of the strain=time response of the meniscal samples. The importance of accurate specimen preparation and actual method development are also presented and discussed in detail.
\end{abstract}

Keywords: meniscus; dynamic mechanical analysis; uniaxial tensile testing

\section{Introduction}

The meniscus is the cartilage in between the femur and tibia and its purpose is to transmit loads [1] whilst providing stability, lubrication and nutrition to the femorotibial joint [2]. It is a smooth, elastic, biconcave disc and is an opaque, milky colour due to its high collagen content, high water content and lack of vascularization. A capillary network originating from the surrounding arteries links to the outer 20-30\% of the meniscus [2] known as the red zone due to its slight pink colour. The remaining meniscus is nourished by synovial fluid. An example of the typical microstructure of meniscus when observed under an Atomic Force Microscope (AFM) is shown in Figure 1.
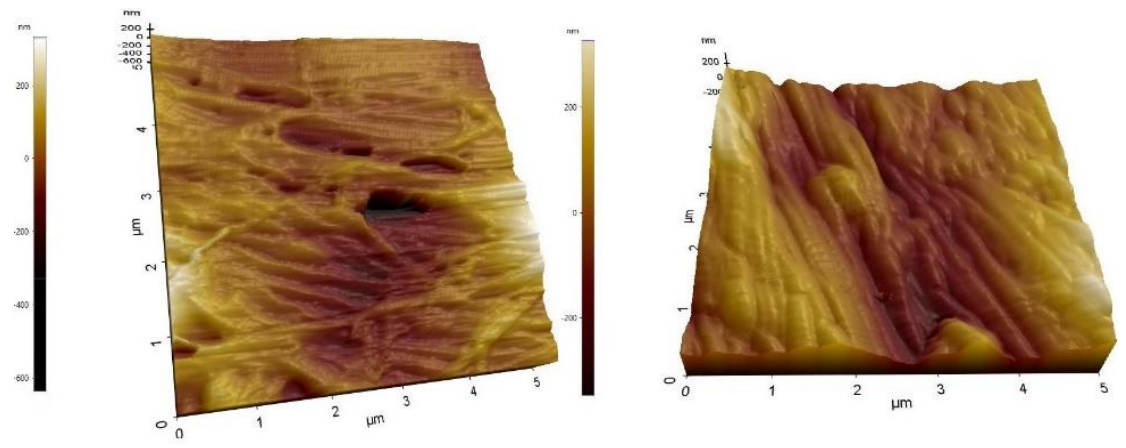

Fig. 1. Tapping mode AFM images of meniscal tissue showing collagen fibres (left) \& (right) channels for synovial fluid flow. 
The causes of meniscal injuries have long been debated however, it is currently thought injuries originate from loading greater than the tissue strength, accumulation of minor injuries, knock-on damage from failing surrounding structures or from muscle fatigue [3]. Surprisingly, these injuries occur commonly throughout the population not solely athletes [4]. Due to the lack of vascularization within the meniscus, the meniscus will not heal itself and treatment is consequently vital to prevent the continued degeneration further injuring and causing pain to a person.

Treatment previously involved removal [3] however, this leads to loss of full mobility in the short term and an increased likelihood of osteoarthritis and narrowing of the joint's space in the long term [2]. Therefore, recent treatment has evolved to "preservation, repair or replacement" [5], although research suggests the mechanical qualities of both replacements and repaired menisci diminish overtime [5], contrary to a healthy, undamaged meniscus.

In this study we have adopted a number of Engineering Mechanics approaches to study the in vivo properties of menisci to both generate knowledge in a relatively unresearched field, as well as to inform clinicians on how meniscal replacements must be designed to better mimic a healthy meniscus and ultimately improve a patient's quality of life. Under normal loading in vivo, the meniscus is subject to a vertical compressive force; whereas through circular traction, this results in a tensile force in the circumferential direction, along which most of the collagen fibres are orientated [6]. Therefore, the methods proposed to study the resulting meniscal behaviour include uniaxial tensile tests and Dynamic Mechanical Analysis (DMA).

\section{Materials and methods}

\subsection{Uniaxial tensile testing}

Whole porcine menisci were sliced into layers normal to the vertical axis using a method outlined by Bonomo et al [6]. This begins with the menisci being defrosted at room temperature and placed in a cavity in an extruded polystyrene (XPS) block, with the tibial surface nearest to and parallel with the edge of the block. The cavity is then filled with Polyfreeze (Sigma-Aldrich, United States). The meniscus, Polyfreeze and XPS is then submerged in liquid nitrogen for two minutes until frozen. The assembly is then thinly sliced normal to the vertical axis using an MBS 240/E Micro Band Saw (Proxxon, Germany). A guide is securely fastened at the desired distance from the blade. The XPS block can then be run along the guide and thinly sliced, as shown in Figure 2a. The 14-teeth-per-inch blade must be on a fast setting (approximately $250 \mathrm{~m} / \mathrm{min}$ ), and the sample pushed through the blade at a slow and steady speed: approximately $10 \mathrm{~mm} / \mathrm{s}$. If the sample is pushed through the blade too fast, the saw can get caught in the frozen sample and stop. Alternatively, if the sample is pushed too slowly, the section of the sample that has been cut can refreeze together before the rest of the sample is cut. As slices of $0.5 \mathrm{~mm}$ thickness or less produce variable results due to artificially high or low fibre fractions [9], thicknesses were used in the range 0.7 to $1.1 \mathrm{~mm}$. As the layers defrost, the polystyrene and PolyFreeze can be mostly removed with tweezers, leaving the meniscal slices. Slices are then submerged in PBS to remove any remaining PolyFreeze. The band saw provides an inexpensive alternative to cryosectioning that can produce thicker sections, while providing more accurate cuts than a scalpel.

Dog-bone shaped samples from desired regions can then be stamped from the slices using a custom-made stamp from Ray-Ran Test Equipment (Ray-Ran, UK). The stamp can make accurate, repeatable, and smooth cuts that ensures all samples have the same dimensions, which are shown in Figure $2 \mathrm{~b}$. Samples are fully hydrated immediately before being stamped to minimize dimension changes that occur as the samples dry. The relatively large sample width is used to minimise the effect of sectioned fibres in a sample. While there is still potential for some sectioning along the sides of the sample, this should be a low proportion of total fibres contained. Samples were taken from a variety of depths into the meniscus and from red-red, red-white and white-white regions across the central body and the two horns.

Whole menisci and dog-bone samples are preserved prior to testing by freezing. It has been found that freezing the samples does not alter the mechanical properties of soft tissues [7]. Samples are also hydrated with Phosphate Buffered Saline (PBS), a salt solution, during testing. In vivo, the meniscus is submerged in synovial fluid, a lubricating, nonNewtonian fluid. PBS has been shown to not significantly affect the mechanical properties in comparison to synovial fluid [8]. 


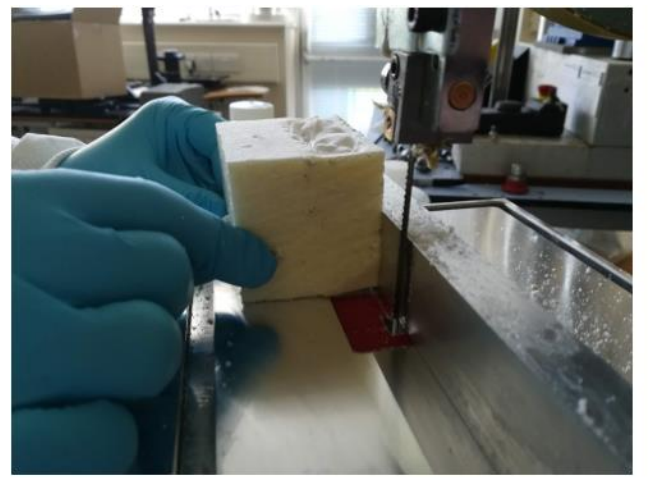

(a)

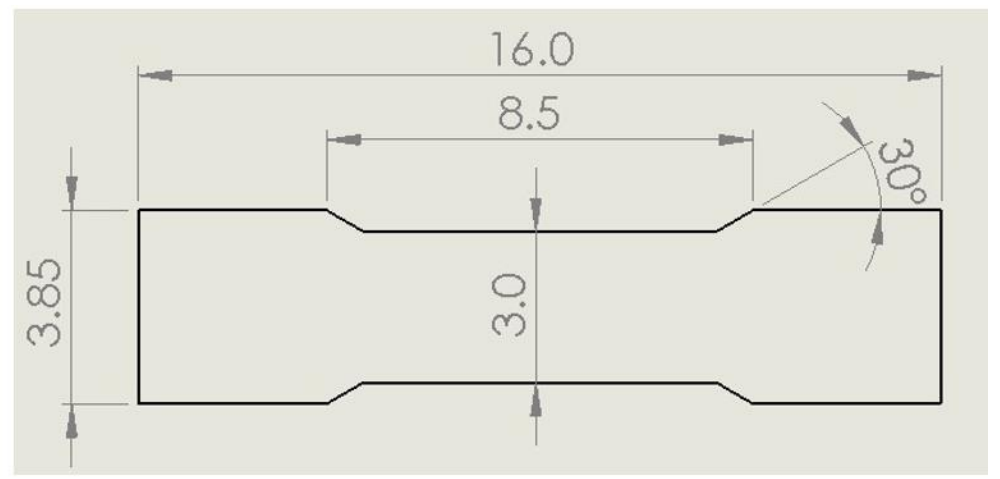

(b)

Fig. 2 Meniscus sample in PolyFreeze and XPS being sliced(a) \& (b) dimensions of the dog-bone shape in mm

For the uniaxial tensile testing of a sample and a Deben Tensile Rig (Deben, UK) with a 200N load cell was used. Immediately before testing, a black speckle was applied to the upper surface of the sample using an airbrush to enable Digital Image Correlation (DIC) for strain measurements. The dog-bone sample was then placed on the rig, where two clamps grip either side of the sample, with an initial distance between clamps of $10 \mathrm{~mm}$. This means the clamps grip only the large ends of the dog-bone shape. The clamps consist of steel blocks with a serrated edge that are screwed into the rig, which has a smooth surface. Thus, the samples are gripped on each side between one serrated surface and one smooth surface. The serrated surface allows the clamp to better grip into the soft and elastic sample. Samples are clamped in a relaxed state, although some slack is introduced to the sample during clamping causing a small compressive force to register. The $10 \mathrm{~mm}$ extension is then slightly largened to reach a zero-stress state.

Using the accompanying Deben software, the rig can then be programmed to perform a chosen test. While tests to fracture can be performed, this method aims to investigate the behaviour of the meniscus under in vivo loading, so fracture was not required. Instead, the test used involves a constant rate extension to approximately $15 \%$ strain, followed by relaxation test that is can be terminated at a desired time. This method results in the full pre-yield stressstrain curve being observed, without significant yield occurring. The relaxation test then provides time-dependent information from the same test, allowing for analysis of viscoelastic properties. The Deben rig offers 5 extension rates, ranging from $0.1 \mathrm{~mm} / \mathrm{min}$ to $1.5 \mathrm{~mm} / \mathrm{min}$. The Deben software records the force across the sample, extension, and time elapsed, plotting it in real time during tests and allowing for data export afterwards. The sample can be preconditioned prior to testing if desired. A camera must also take periodic images of the sample from above as the test takes place. A suggested minimum rate is 10 images per $1 \%$ strain.

To analyse the data from the Deben software after the test, the engineering stress must be calculated from the force readings by dividing the recorded force by the initial cross-sectional area. The engineering or nominal strain can be calculated from the displacement given by Deben: the current extension divided by the initial length of the sample at the zero-stress state. This assumes that the strain is constant throughout the sample. In the case of a complicated structure like meniscus, it is unlikely that strain is constant. Additionally, it provides no information on the strain in the transverse direction. It has been found in many studies that the meniscus exhibits a very high Poisson ratio under plane stress, depending on the sample's location in the meniscus. For this reason, it is important to have a method to measure strain throughout the sample surface. DIC can be performed with the images taken of the samples speckled surface. Software analyses the images along with an inputted base dimension, tracking blocks of pixels to obtain a 2D strain field of the sample. The software DaVis (LaVision, Germany) was used to perform DIC. By selecting two coaxial points on the surface, the program can produce a plot of strain against time. The time can be used with that from the Deben software to correlate the DIC strain with the equivalent stress measured in the sample, allowing for the plotting of stress-strain data. The sample experiences a contraction strain in the transverse direction which can also be measured using DIC, allowing for the Poisson's ratio to be calculated. A stress-time plot can be used to fit an exponential approximation to the relaxation data, from which the characteristic times of relaxation can be calculated. 


\subsection{Dynamic Mechanical Analysis (DMA)}

For the DMA compression tests the initially frozen meniscal samples were thawed for 30 minutes at $35{ }^{\circ} \mathrm{C}$ to prevent denaturing of any remaining cells (which denature above $37^{\circ} \mathrm{C}$ ) and to allow ease and non-warping of cutting. Meniscal cylinders were then cut perpendicular to the tibial surface using a $5.9 \mathrm{~mm}$ diameter biopsy punch, to ensure simulated loading was in the same direction as in vivo loading, and care was taken to ensure these contained solely one meniscal region (posterior, anterior or mid body) and no ligaments. A diameter of $5.9 \mathrm{~mm}$ was chosen, as it is small enough to allow one sample per meniscal region and large enough to not fall during testing. Cylinders were cut coplanar to the tibia-facing surface using a sharp and sterile blade to ensure the upper surface area touching the DMA was equal across all samples and equal to the lower surface receiving the reaction force.

Samples were placed in small containers, immersed in Phosphate-Buffered Saline (PBS) solution (the pH of PBS is 7.4 and so mimics the $\mathrm{pH}$ of blood [9]) and stored at $4{ }^{\circ} \mathrm{C}$. Each meniscus was separately defrosted, and biopsy punched prior to each DMA run to ensure they remain fresh throughout the test. A $15 \mathrm{~mm}$ thin aluminium plate containing a $6.5 \mathrm{~mm}$ diameter hole, shown in Figure $3 \mathrm{~b}$ was fabricated to prevent cylindrical samples from tipping during testing and so invalidating a test. The hole was large enough to fit samples and prevent them from tipping, yet thin enough not to alter the meniscal surface area available to PBS re-entering after having been pushed out during a loading cycle.

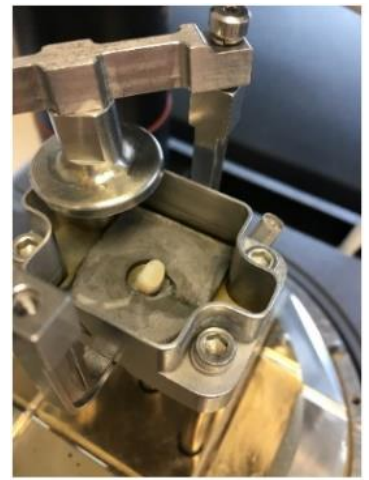

(a)

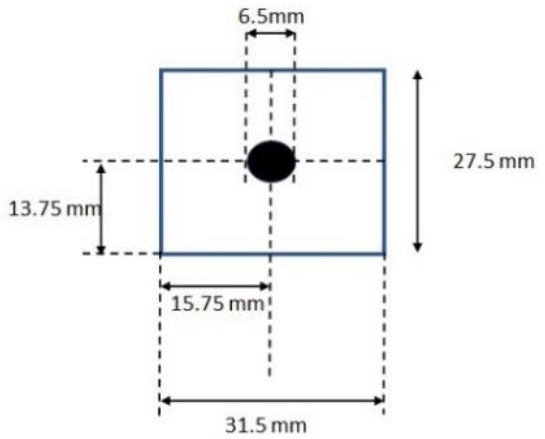

(b)

Fig. 3 Meniscus sample and aluminium plate within the submersion clamps of the DMA (a) \& (b) schematic diagram of the aluminium plate.

After mass, zero and stiffness calibrations, samples were loaded into the dry submersion clamp of the DMA (Q800 TA Instruments), the compression clamp was secured above the centre of the sample and lowered at a pre-load force of $0.03 \mathrm{~N}$. This value was chosen, as it was just enough to overcome the surface tension of the PBS solution whilst not being so high to introduce compression. This ensured the compression clamp was sufficiently strong to re-enter the PBS after each cycle, so the cylinder did not tip, and cyclic loading could occur. The submersion clamp was filled with PBS solution after the pre-load force had been applied to allow precise placement of the sample without viscous forces. The furnace of the DMA was closed, and temperature set to $37^{\circ} \mathrm{C}$ to mimic body temperature.

The first stage of the DMA method input was the equilibration stage where the temperature was held at $37^{\circ} \mathrm{C}$ and the pre-load force at $0.03 \mathrm{~N}$ for 5 minutes. This ensured the whole sample had reached the set temperature, as different temperatures may vary elasticity, and to give the sample time to settle to a constant height. The height after 5 minutes was recorded as the initial height and this was used to calculate the change in strain during the loading stage.

The second stage of the DMA method input was the cyclic loading stage where samples were subjected to rapidly applied and removed ramp forces to simulate the varying loading and unloading felt by the knee during a run. This involved applying a force ramp from the pre-load force $0.03 \mathrm{~N}$ to the maximum force $\left(\mathrm{F}_{\max }\right)$ and then a force ramp down from $F_{\max }$ to the pre-load force $0.03 \mathrm{~N}$. This was repeated 6000 times at a force application rate of $250 \mathrm{~N} / \mathrm{min}$.

$F_{\max }$ was found to be $1.914 \mathrm{~N}$, by using the stress per unit area $(\sigma)$ expected during activities on the meniscus of $0.07 \mathrm{MPa}$ [10], a surface area of the base of the meniscal cylinder (A) and a sample diameter of $5.9 \mathrm{~mm}$. A slightly higher $\mathrm{F}_{\max }$ of $2.00 \mathrm{~N}$ was chosen to account for the extra impact felt by the meniscus during running compared to normal activities and a ramp was used rather than holding a force for a set time because the maximum hold time lead to a frequency too slow to be considered running. Using a ramp to vary between $0.03 \mathrm{~N}$ and $2.00 \mathrm{~N}$ meant the force 
varied by $1.97 \mathrm{~N}$ per ramp. A force application rate of $250 \mathrm{~N} / \mathrm{min}$ meant the ramp took 0.47 seconds to vary from 0.03 $\mathrm{N}$ to $2.00 \mathrm{~N}$ and so, a full cycle took double this which was 0.95 seconds $(1.06 \mathrm{~Hz})$.

The average moderate running pace is 130 steps per minute [11] equating to a loading frequency of $2.16 \mathrm{~Hz}$. As running is undertaken by 2 menisci, the average moderate running frequency of $2.16 \mathrm{~Hz}$ per 2 may be equated to 1.08 $\mathrm{Hz}$, so simulating a frequency of $1.06 \mathrm{~Hz}$ is accurate.

The final stage of the DMA method input was the recovery stage where the force was held at the pre-load force of $0.03 \mathrm{~N}$ and temperature of $37^{\circ} \mathrm{C}$. The change in height and strain were recorded for between 3 and 8 hours depending on the availability of the DMA. The sampling interval was placed at the maximum value of $0.1 \mathrm{~s} /$ point during the cyclic loading phase and at a lower value of $2.0 \mathrm{~s} /$ point during the recovery phase to ensure the most accurate response was recorded during the phase the sample was changing the most (i.e. cyclic loading phase) whilst ensuring not too much data was collected in the unchanging phase (i.e. recovery phase).

\section{Results and discussion}

\subsection{Uniaxial tensile testing}

Figure 4 shows the typical stress-strain and stress-time curves obtained during tensile testing of the meniscus samples. The stress-strain curve shows the results using the strain calculated from the rig (in blue) and from DIC (in black). Both curves demonstrate an expected toe region followed by a more linear region. Only the blue curve exhibits post yield behaviour with the softening. This can be explained by the presence of stress concentrators near the clamps. DIC revealed higher strains at these positions relative to the middle sections, which could arise from complicated interactions between a variety of factors. The clamps inhibit the small movement of fibres sliding relative to each other that may occur in the central regions, as well as stopping any contraction strain near to the clamp in the transverse direction. The clamps are also close to the transitional region of the sample where the width narrows. As the DIC strain above was taken from the middle regions, where these factors have less impact, the strain is lower than that measured from the rig which includes the full length of the sample. This demonstrates the importance of using DIC to analyse strain. Thus, using the DIC strains, the full and accurate pre-yield behaviour can be shown using this method, with the sample dimensions allowing for sample to be taken from distinct regions of the meniscus. This method can thus be used to fully characterise the tensile behaviour in the meniscus. Additionally, the stress-time curve demonstrates the considerable relaxation that occurs due to the viscoelasticity of the fibres. For the data in Figure $4 \mathrm{~b}$, the MATLAB curve fitting toolbox was used to fit a single-term exponential. The results indicated a characteristic relaxation time of $43.3 \mathrm{~s}$.

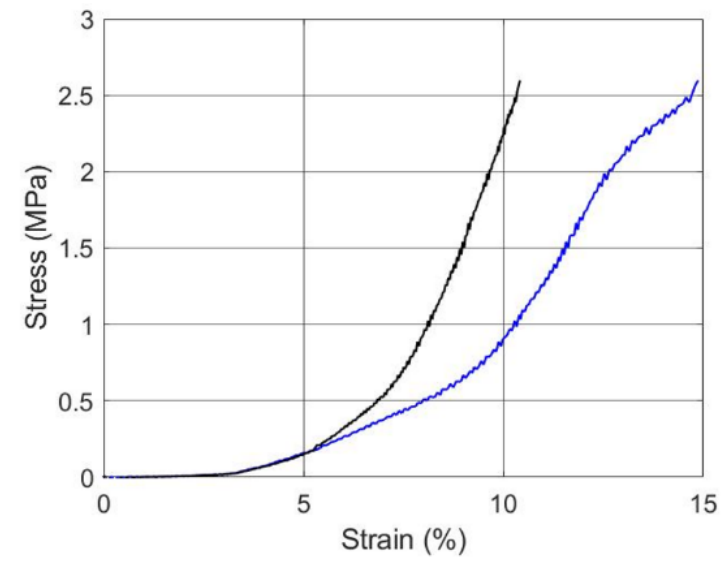

(a)

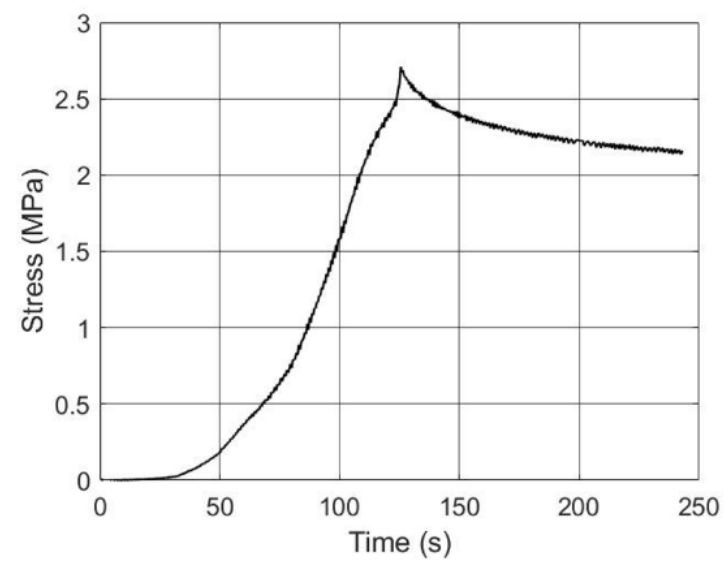

(b)

Fig. 4 Typical stress-strain curve using strain calculated from the rig (blue) and DIC (black) (a) \& (b) typical stress-time curve

Potential sources of error in this test include the sample slipping from the clamp under loading. This was found to occur very rarely with hydrated samples, and due to the dog-bone shape of the sample and any artefacts left on the surface from the slipping, it is easy to spot when it does occur so the results can be discarded. The issue of the presence 
of stress concentrators can be overcome using the DIC measurements, however, there is also a possibility of this leading to early fracture at or near the clamps. This was observed to happen very infrequently, and when it does occur it is very evident both visually and, in the results, with a sharp fall in stress, meaning that the results are known to be erroneous. It is possible that increasing the width of the dog-bone ends slightly may reduce these stress concentrators and should be investigated. Analysis of the strain fields across the sample do show unevenness in strain even in the central regions of the sample. This can be attributed to the method of loading in the sample, with fibres bearing the large majority of the load with the interstitial matrix having a much lower stiffness. As the fibres independently bear load, the matrix can be stretched in the space between them to higher strains, especially if there is a small deviation between the sample axis and the fibre orientation that causes larger shear stresses. To overcome this, average strain can be taken across the whole sample as opposed to solely measuring strain along one line.

\subsection{Dynamic Mechanical Analysis (DMA)}

Figure 5 shows the typical strain-time response from the meniscal samples during the walking and running DMA simulations. The first 5 minutes are the acclimatization period, the next approximately 70 minutes the loading period and the remaining time corresponds to the recovery period. Generally, samples recovered significantly during a cycle as their loading lines, corresponding to forces being applied and removed, are relatively thick. Even after a long recovery period, no sample recovered to its initial height implying the DMA test may not necessarily give a full representation of in vivo conditions and that an extra force, such as the pressure around the interstitial joint or from living glycosaminoglycans attracting water molecules, fully recovers the meniscus. Glycosaminoglycans are chains that attract and hold water and so are temporarily resistant to load, due to water's incompressibility, before water flows out and therefore their purpose is to keep the extracellular matrix (ECM) hydrated, as well as to provide the bearing capacity against compressive forces [2].

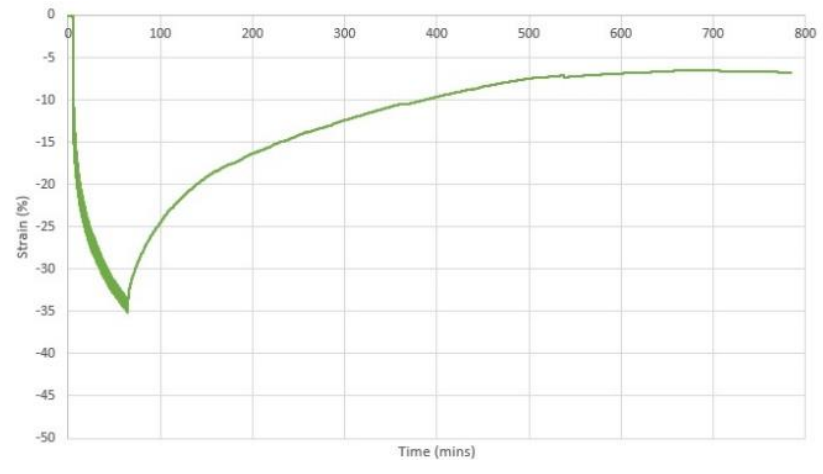

(a)

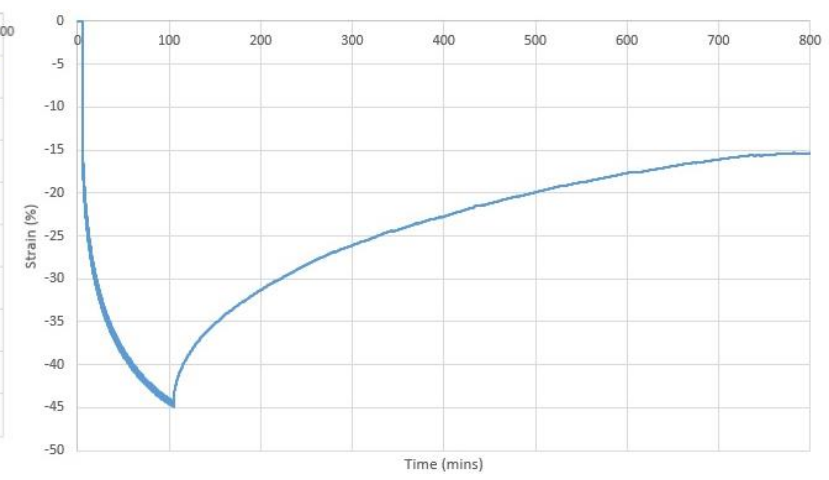

(b)

Fig. 5 Typical strain-time curves obtained from a walking (a) \& (b) running DMA simulation.

In addition, and in the case of the running simulations, it can be suggested that the existing network of water channels may provide an additional mechanism that would allow energy transfer from the solid to the liquid phase when a force is applied. This mechanism is only evident if the loading frequency is fast enough, so that water has no time to slowly leave the network and instead, is momentarily stationary and incompressible. This, in turn means, that the interstitial fluid rather than the meniscus bears the impact and so the structure is protected from sudden loading.

\section{Conclusions}

In summary, we have been able to successfully develop and implement two micromechanical experimental approaches to study the mechanical behaviour of meniscal tissue. It was demonstrated that both uniaxial tensile testing and DMA compression testing methodologies generate meaningful results that provide information on stress-strain non-linearity in addition to time-dependent viscous effects. It is strongly believed that the introduced preparation techniques would allow for precise samples to be taken from various regions of the meniscus, thus enabling 
investigation into the variation in mechanical in this complex biological system. This research forms part of a much larger collaborative effort aimed at understanding and enhancing our fundamental understanding of meniscus and to enable scientists across the field to improve current techniques and ultimately improve the quality of life of the many people affected.

\section{Acknowledgements}

O.B would like to acknowledge the European Union's Horizon 2020 -EU.1.3.2. - Nurturing excellence by means of cross-border and cross-sector mobility under the Marie Skłodowska-Curie individual fellowship MSCA-IF-2017, MetaBioMec, Grant agreement ID: 796405.. The authors would also like to thank Marzena Tkaczyk and Nick Hawkins for their help and support throughout this project.

\section{References}

[1] K. Dragnevski, M. Tkaczyk and V. Vetri, “Advanced microscopy analysis of the micro-nanoscale rchitecture of human menisci," Scientific Reports, vol. 9, no. 1, 2019.

[2] A. Bedi, S. Rodeo and A. Fox, "The Basic Science of Human Knee Menisci: Structure, Composition and Function," Sports Health, vol. 4, no. 4, pp. 340-351, 2012.

[3] J. Matyas, L. Barclay and J. Rattner, "New understanding of the complex structure of knee menisci: Implications for injury risk and repair potential for athletes," Scandinavian Journal of Medicine and Science in Sports, vol. 21, no. 4, pp. 543-553, 2011.

[4] D. Sohn and C. Moorman, "Meniscal Debridement," Journal of Knee Surgery, vol. 21, no. 2, pp. 145-153, 2008.

[5] S. Caridade, A. Frias and H. Pereira, "Biomechanical and cellular segmental characterization of human meniscus: building the basis for Tissue Engineering therapies," Osteoarthritis Research Society Vol. 22 No. 9, pp. 1271-1281, 2014.

[6] F. P. Bonomo, J. J. S. Gregory and O. Barrera, “A procedure for soft heterogeneous and irregular-shaped tissue precise slicing,” Under Review, 2019.

[7] J. Virues Delgadillo, S. Delorme, R. El-Ayoubi, R. DiRaddo and S. Hatzikiriakos, "Effect of freezing on the passive mechanical properties of arterial samples," Journal of Biomdeical Science and Engineering, vol. 3, pp. 645-652, 2010.

[8] E. Lakes, C. Kline, P. McFetridge and K. Allen, "Comparing the mechanical properties of the porcine knee meniscus when hydrated in saline versus synovial fluids," Journal of Biomechanics, vol. 48, no. 16, pp. 4333-4338, 2015.

[9] J. A. Kellum, "Determinants of blood pH in health and disease," Critical Care, vol. 4, no. 1, p. 6-14, 2000.

[10] A. Seitz, F. Galbusera and C. Krais, "Stress-relaxation response of human menisci under confined compression conditions," Journal of the Mechanical Behavior of Biomedical Materials, vol. 26, pp. 68-80, 2013.

[11] G. Burns, J. Zendler and R. Zernicke, "Step frequency patterns of elite ultramarathon runners during a 100-km road race," Journal of applied physiology, vol. 126, no. 2, pp. 462-468, 2019. 